\title{
Failure Diagnosis System for a Ball-Screw by Using Vibration Signals
}

\author{
Won Gi Lee, ${ }^{1}$ Jin Woo Lee, ${ }^{1}$ Min Sung Hong, ${ }^{1}$ Sung-Ho Nam, \\ YongHo Jeon, ${ }^{1}$ and Moon G. Lee ${ }^{1}$ \\ ${ }^{1}$ Department of Mechanical Engineering, Ajou University, San 5, Woncheon-dong, Yeongtong-gu, Suwon 443-749, Republic of Korea \\ ${ }^{2}$ Korea Institute of Industrial Technology (KITECH), 1271-18, Sa-3-dong, Sangrok-gu, Ansan 426-791, Republic of Korea
}

Correspondence should be addressed to Moon G. Lee; moongulee@ajou.ac.kr

Received 1 April 2015; Accepted 21 June 2015

Academic Editor: Carlo Trigona

Copyright (C) 2015 Won Gi Lee et al. This is an open access article distributed under the Creative Commons Attribution License, which permits unrestricted use, distribution, and reproduction in any medium, provided the original work is properly cited.

Recently, in order to reduce high maintenance costs and to increase operating ratio in manufacturing systems, condition-based maintenance $(\mathrm{CBM})$ has been developed. CBM is carried out with indicators, which show equipment's faults and performance deterioration. In this study, indicator signal acquisition and condition monitoring are applied to a ball-screw-driven stage. Although ball-screw is a typical linearly reciprocating part and is widely used in industry, it has not gained attention to be diagnosed compared to rotating parts such as motor, pump, and bearing. First, the vibration-based monitoring method, which uses vibration signal to monitor the condition of a machine, is proposed. Second, Wavelet transform is used to analyze the defect signals in time-frequency domain. Finally, the failure diagnosis system is developed using the analysis, and then its performance is evaluated. Using the system, we estimated the severity of failure and detect the defect position. The low defect frequency $(\approx 58.7 \mathrm{~Hz})$ is spread all over the time in the Wavelet-filtered signal with low frequency range. Its amplitude reflects the progress of defect. The defect position was found in the signal with high frequency range $(768 \sim 1,536 \mathrm{~Hz})$. It was detected from the interval between abrupt changes of signal.

\section{Introduction}

Today's manufacturing systems have become gradually automated for the purposes of productivity improvement and cost savings. So, the production facilities are to be operated for 24 hours. However, the full ramp-up of automated production lines with high operating ratio has also caused various mechanical failures and, accordingly, increased economic losses. Generally, about 15 to $40 \%$ of entire production costs are consumed for equipment maintenance, and $30 \%$ of the maintenance costs are lost for unnecessary or improper job [1].

Recently, in order to save high maintenance costs and to increase manufacturing system's operating ratio, conditionbased maintenance (CBM) method has been developed $[2,3]$. Unlike traditional maintenance that needs to be carried out on a regular time basis, the CBM detects any system defect early and identifies the cause of failure efficiently through use of indicator signals that reflect the actual operation condition of systems and accordingly reduces unnecessary costs and maintenance efforts $[4,5]$. In order to realize the CBM method effectively, it is needed to identify the physical and structural characteristics of equipment, to select the appropriate indicator signals that show the status of facilities and to evaluate the condition of them. For this process, accurate analysis of equipment pieces and their failures, appropriate monitoring of indicator signals, correct diagnosis of defect signals, and proper alarm of the condition are required.

Research to enhance maintenance efficiency has long been conducted for diverse mechanical elements and modules. Maslach [6] monitored and analyzed the condition of a lubricated bearing, one of the representative mechanical elements, and Spoerre [7] applied the analysis to discover and classify bearing failures. Moreover, Schoen et al. [8] identified defects in motor bearings through use of current signals in motors, and Autar [9] implemented an expert system to diagnose the condition and failure of diesel engines. 
Most research has been conducted on rotating elements such as engines, turbines, motors, gears, shaft, or compressors, for which it is easy to measure and treat signals. Recently, research on linearly reciprocating modules that creates nonperiodical signals with abrupt change is also making meaningful headway. However, since studies on linearly reciprocating components have been limited to engines or compressors, further research needs to be directed at diverse linearly reciprocating modules.

In this study, a defect detection and diagnosis method was proposed for the ball-screw-driven stage, a representative linearly reciprocating mechanical module, and a failure diagnosis system was designed and applied through the use of CBM. For this process, firstly, normal and defect frequencies were derived from the common failure of the ball-screw. Second, a vibration signal acquisition module was implemented in the diagnosis system. The normal and defect signals were obtained and then the derivation was investigated. Also the amplitude change of defect signal was found according to the progress of failure. Third, we applied Wavelet transformation to the signal so that the system identifies the defect frequencies and estimates their progress. The system is stand-alone and PC-based one so that it can be applied to various manufacturing equipment pieces or facilities.

\section{Defect Frequencies of Ball-Screw}

Most mechanical systems generate vibration signals when they are operated, signals which offer diverse and plentiful information on the condition of systems. In particular, when the system runs continuously, that is, in steady state, the various frequency components of vibration signals represent different characteristics pertinent to each mechanical component, and the amplitude of frequency components varies according to the condition of system operation [10]. In this study, vibration was selected as indicator signals to detect and diagnose the defects of ball-screw. In addition, defect frequencies were derived for main failure modes, and a vibration monitoring method was presented using the frequencies.

2.1. Failure of Ball-Screw. As shown in Figure 1, the ball-screw consists of steel balls, screw shaft, nuts, seals, and tube. The steel balls between the nuts and screw shaft roll so that the nut moves linearly along the screw shaft when the shaft is rotated by an actuating motor [11]. Usually, the nut carries external load. Therefore, while the steel balls are rotated by the actuation under the external load and friction, the grooves in the screw shaft are under repeated compressive stress, which causes fatigue in the screw shaft, and then various failures detected in the ball-screw.

Autar identified that defects such as fatigue, fractures, and strains are mostly found in the parts that are subject to rolling friction and that the defects occurring in screw shafts and steel balls represent major failure modes [9]. Figure 2 shows flaking, a representative fatigue fracture where the surface of grooves is exfoliated by the repeated compressive stress.

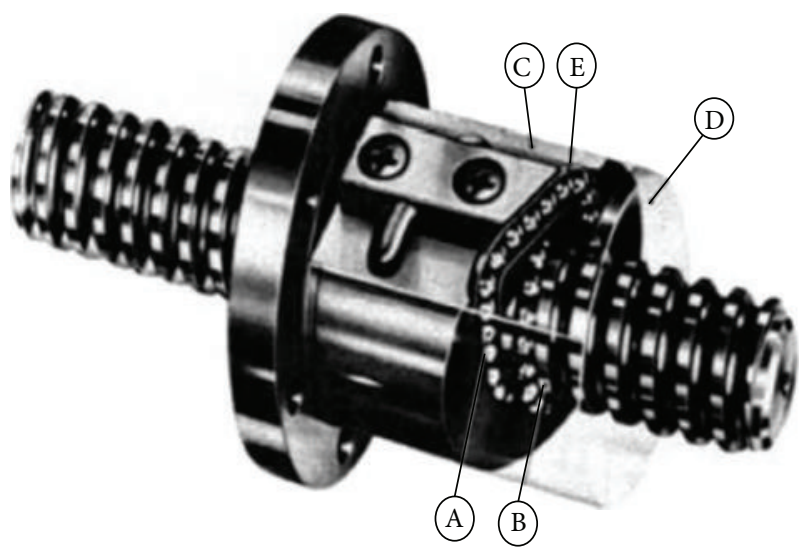

$\begin{array}{ll}\text { A: steel ball } & \text { D: seal } \\ \text { B: screw shaft } & \text { E: tube } \\ \text { C: nut } & \end{array}$

FIGURE 1: Structure of ball-screw.

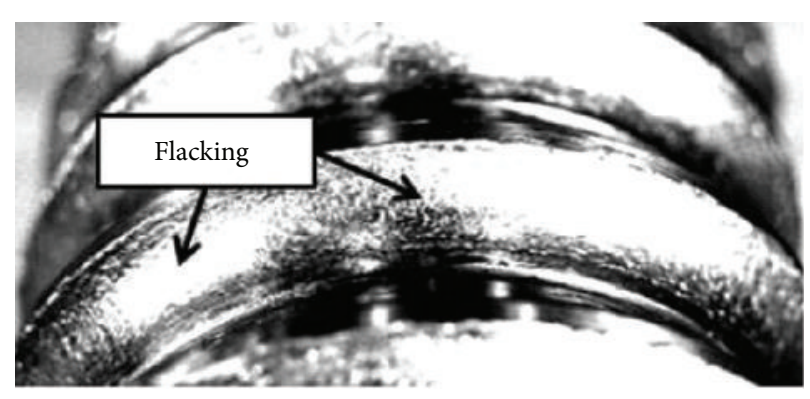

FIGURE 2: Example of fatigue failure: flacking.

2.2. Defect Frequencies. Fatigue fractures, the major defect of the ball-screw, start with local small defects but finally break the mechanical module. During the progress, the defect signal is distorted more from the normal. After a small defect occurs on the surface of the screw shaft which is in contact with the steel balls, shocks take place regularly and periodically whenever the steel balls pass through the faults. The period of the shocks varies according to the location of the defects in the components of the ball-screw. For this reason, different defect frequencies occur for each component. Harris and McCool [12] detected defects in rolling bearing by use of these defect frequencies.

In this study, we propose a new method to derive defect frequencies from the relative speed of components of the ballscrew, through use of the findings of Harris and $\mathrm{McCool}$ and by assuming the ball-screw as a rolling bearing. The balls are rotating, revolving, and sliding in the inner and outer races. The inner race is male screw nut and outer one is female nut. When the balls pass along the gap between the inner and outer races, the motion generates each typical vibration. More details can be found in Harris and McCool [12].

If Harris method is applied, the defect frequencies are deduced considering the nut as an outer ring and the screw as an inner ring as follows. When the contact angle made by the ball, nuts, and screw shaft is $\alpha$, the pitch diameter of balls 


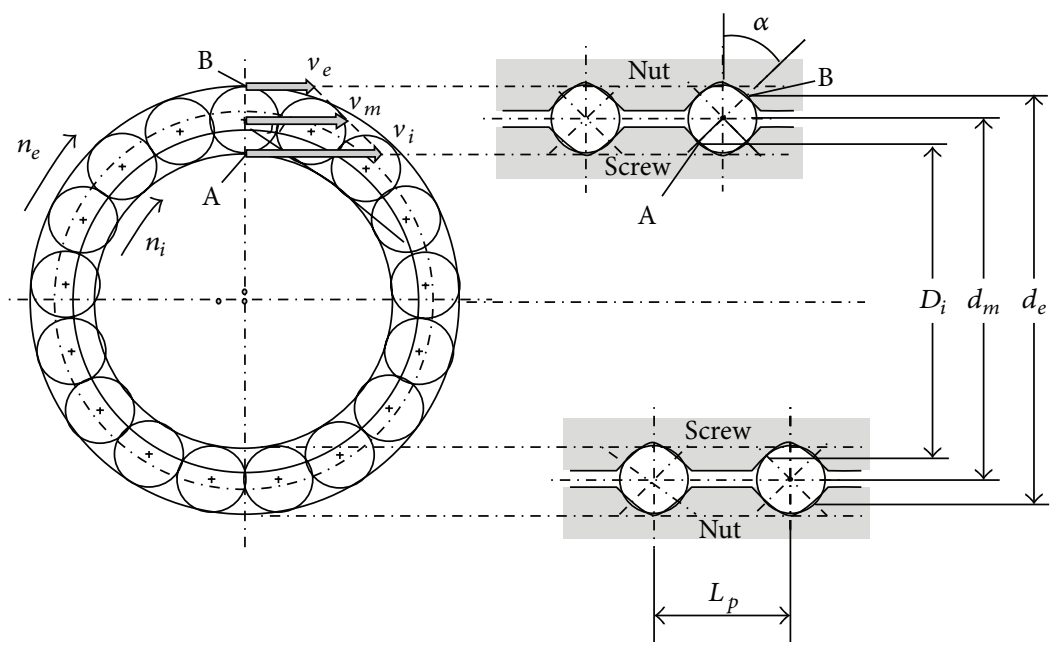

FIGURE 3: Modeling ball-screw based on rolling bearing.

is $d_{m}$, the diameter of each ball is $D_{w}$, the rotational speeds of external and internal races are $n_{e}$ and $n_{i}$, and the number of balls is $z$, the defect frequencies are derived as shown in (1) to (3) from Figure 3. For the speed of rotation, the nut does not have any speed $\left(n_{e}=0\right)$ but the screw rotates $\left(n_{i}=n\right)$. Each defect frequency is ball pass frequencies of shaft (BPFS), ball pass frequency of nut (BPFN), and ball spin frequency (BSF). Among the frequencies, the BPFS shown in (1) constitutes the major vibration signal because it has the largest amplitude and mostly appears in low frequency domain. Note that the rotations speeds are in $\mathrm{rpm}$ and the frequencies are in $\mathrm{Hz}$ :

$$
\begin{aligned}
\mathrm{BPFS} & =\frac{1}{120} z n\left(1+\frac{D_{w}}{d_{m}} \cos \alpha\right), \\
\mathrm{BPFN} & =\frac{1}{120} z n\left(1-\frac{D_{w}}{d_{m}} \cos \alpha\right), \\
\mathrm{BSF} & =\frac{1}{120} n \frac{d_{m}}{D_{w}}\left(1-\frac{D_{w}}{d_{m}} \cos \alpha\right)\left(1+\frac{D_{w}}{d_{m}} \cos \alpha\right) .
\end{aligned}
$$

However, the findings of the above three equations are still not for ball-screw but rotational rolling bearings. Since defect frequencies of the ball-screw are required to be newly derived, we introduced the effective pitch diameter of ball $d_{m}^{\prime}$ instead of the pitch diameter of ball $d_{m}$ and the effective number of balls $z^{\prime}$ instead of the number of balls $z$. They are drawn from (4) from Figure 4:

$$
\begin{aligned}
d_{m}^{\prime} & =\left(L_{p}^{2}+\left(\pi D_{b}\right)^{2}\right)^{1 / 2}, \\
z^{\prime} & =\frac{d_{m}^{\prime}}{D_{w}}
\end{aligned}
$$

Because the equation has $\cos \alpha$ in the case that the contact angle of the grooves of balls and the screw shaft is not $0^{\circ}$, the defect frequencies are not the value of integer times of the rotational speed. This makes it easy to analyze frequencies and diagnose defects because the defect vibration frequencies

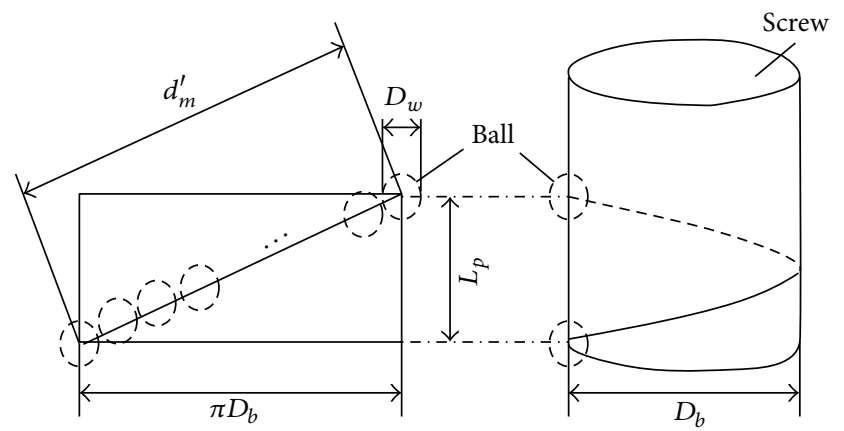

FIGURE 4: Effective pitch diameter and number of balls of ball-screw when considering it a rolling bearing.

are distinguished from the vibration frequency by the shaft rotation and its high harmonics.

\section{Data Acquisition and Defect Signals}

We collected normal and defect vibration signals of the ballscrew-driven stage, identified the defect frequencies from a failure mode presented in Section 2, and analyzed vibration signals. Also it is investigated how the progress of defect is reflected on the amplitude of signal.

3.1. Experimental Setup. In order to analyze the normal and defect signals of the ball-screw-driven stage, an experimental apparatus was set up as shown in Figure 5. The stage consists of AC motor, ball-screw stage, and LM (linear motion) guide. Its size was one-tenth of the actual ball-screw system that is widely used in CNC machine tools and automation systems. Major specifications of the ball-screw-driven stage are shown in Table 1.

In order to measure and analyze the vibration signals of the test rig, a monitoring system was implemented as shown in Figure 6 and Table 1.

The vibration signals of the ball-screw are measured with an accelerometer mounted on the nuts while the stage 
TABLE 1: Specifications of ball-screw-driven stage.

\begin{tabular}{|c|c|c|c|}
\hline Components & Specifications & Value & Model (maker) \\
\hline \multirow{2}{*}{ Stage } & Repeatability & $\pm 0.02 \mathrm{~mm}$ & \multirow{2}{*}{ LP200-SC-600L-70-H-S (LPK) } \\
\hline & Stroke & $600 \mathrm{~mm}$ & \\
\hline \multirow{2}{*}{ Motor } & Rated rotating speed & $3000 \mathrm{rpm}$ & \multirow{2}{*}{ HF-KP73 (Mitsubishi) } \\
\hline & Input source & 3 phases $(60 \mathrm{~Hz})$ & \\
\hline \multirow{5}{*}{ Ball-screw } & Lead & $20 \mathrm{~mm}$ & \multirow{5}{*}{ HTR 2020A (ISSOKU) } \\
\hline & Circuit & 1.5 turns & \\
\hline & Diameter of ball centers & $21 \mathrm{~mm}$ & \\
\hline & Diameter of balls & $3.969 \mathrm{~mm}$ & \\
\hline & Contact angle & $45^{\circ}$ & \\
\hline
\end{tabular}
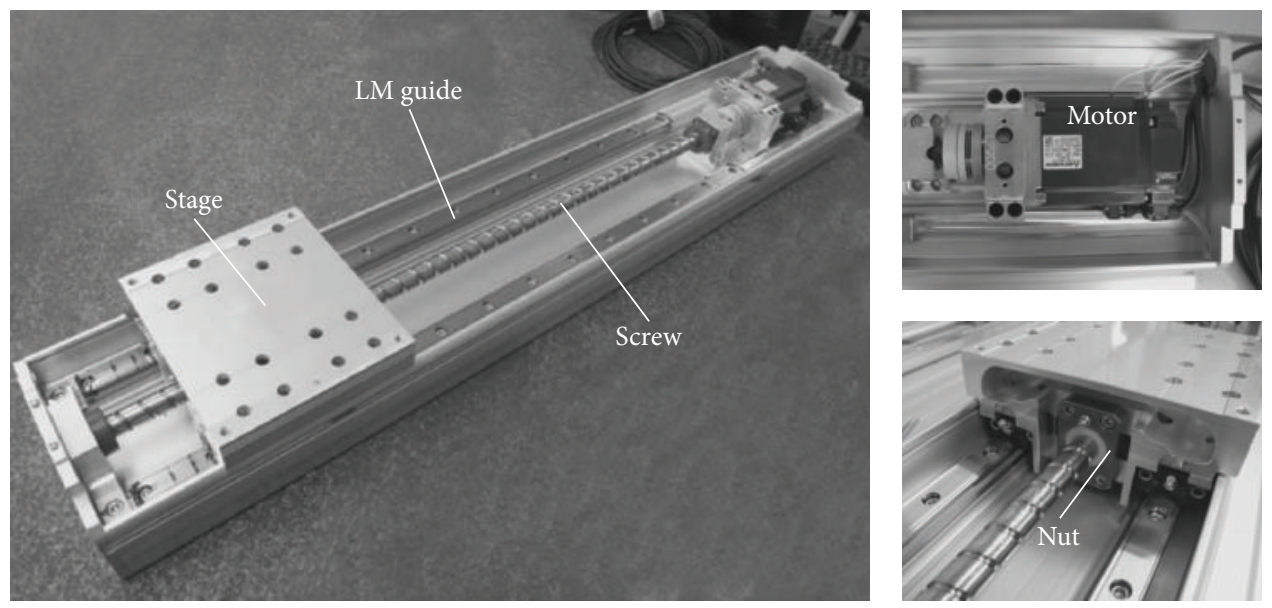

Figure 5: Ball-screw-driven stage.

reciprocates a travel of $267 \mathrm{~mm}$ at a speed of $133 \mathrm{~mm} / \mathrm{s}$ and an acceleration of $5,000 \mathrm{~mm} / \mathrm{s}^{2}$. In order to simulate the defect signals coming from the flaked screw shaft, as shown in Figure 7 , holes with a diameter of $3 \mathrm{~mm}$ were punched on the ball groove with a carbide drill to simulate the defects.

3.2. Normal Signal Analysis. The vibration signals that occurred when the ball-screw-driven stage is reciprocating without defect were analyzed in the frequency domain. As shown in Figure 8, 60 and $120 \mathrm{~Hz}$ were the dominant components in low frequency range. This is because of the electrical current and its harmonics in the motor. In the following analysis, the $60 \mathrm{~Hz}$ component was filtered to clarify the other frequency components. Considering the specifications of the accelerometer, the frequency component less than $1.0 \mathrm{~Hz}$ has no meaningful information.

3.3. Defect Signal Analysis. The defect frequency derived from the flaking of screw shaft used for this study is derived from (1) as follows:

$$
\begin{aligned}
\text { defect frequency } & =\frac{1}{120} z^{\prime} n\left(1+\frac{D_{w}}{d_{m}^{\prime}} \cos \alpha\right) \\
& =0.147 n .
\end{aligned}
$$

Figure 9 shows the change of defect frequency in $\mathrm{Hz}$ according to the rotational speed of the motor $n$ in rpm. The numbers in the left are rotational speeds and the peaks indicated by arrow are the defect frequencies, which indicates that the defect frequencies from (5) took place as predicted. The major electrical noise of frequency, $60 \mathrm{~Hz}$, has been cut off but its second harmonic, $120 \mathrm{~Hz}$, was not filtered out. It appeared as a peak at $120 \mathrm{~Hz}$ in the graphs.

Then, the relation of defect frequency with the number of defective holes was analyzed. As shown in Figure 10, amplitude of the defect frequency tends to be larger when the defect gets worse, which makes it easier to detect and diagnose the defects through defect frequencies and amplitude in steady state.

It has been known that the fatigue strength is saturated after large number of rotations [13]. If the weakened strength is synchronized with the progress of fatigue, it will result in large vibration amplitude. In the experiments, the simulated defect, drilled hole on screw, is mimicking the flacking fatigue. The progress of fatigue was modeled as the number of holes. Therefore, the saturation stands to reason in Figure 10. In the fault estimation of failure diagnosis system, the saturation can be taken into account when considering logarithmic scale of the amplitude progress in Section 5. 

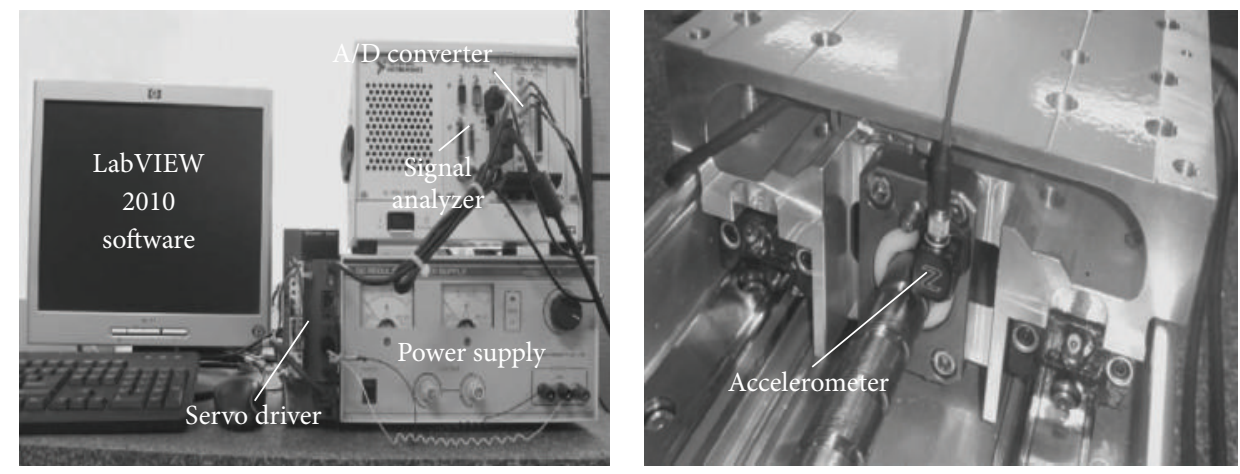

FIGURE 6: Condition monitoring system with data acquisition, sensor, and analyzing S/W.
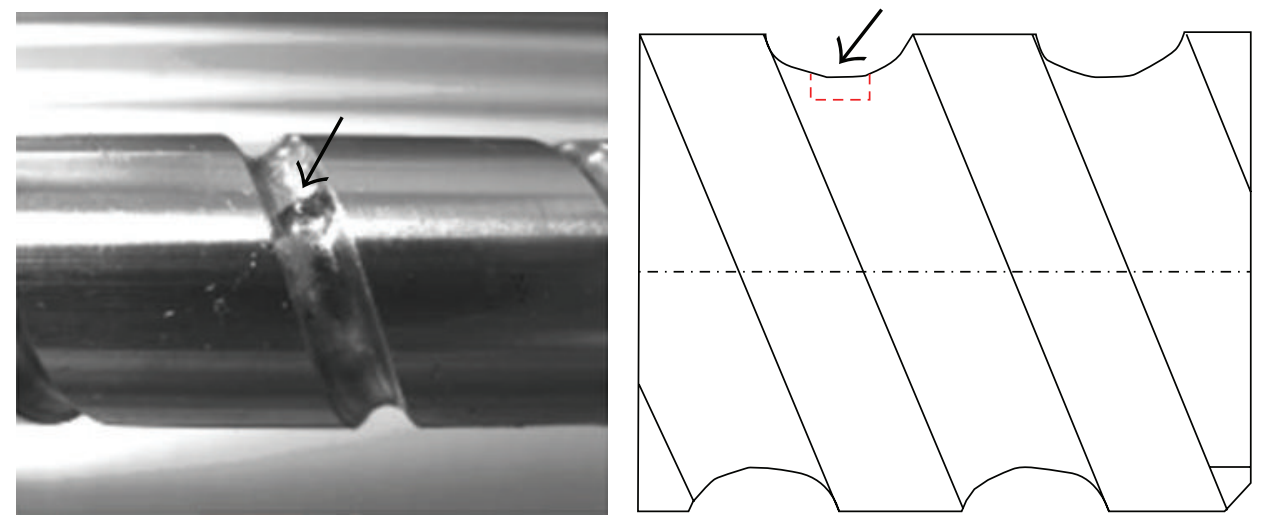

FiguRE 7: Simulated defects (indicated by an arrow).

\section{Time-Frequency Domain Analysis for Ball-Screw}

The defects occurring in the ball-screw generate excessive abnormal signals such as shock signals when the nuts pass through a local defective part. This abrupt change can be detected in time domain. The defect signals from continuous ball-defect interaction can be shown up in frequency domain analysis as in Section 3. Therefore, we indicated the time when the defect signal occurred as well as its frequency components by use of a time-frequency domain analysis, discrete Wavelet transform [12] as in Figure 11.

For more effective analysis, we used the Daubechies Wavelet (db14) function, which is similar to the impulse signals in discrete Wavelet transform. In addition, we analyzed the signals from levels 1 to 9 to restrict the frequency analysis domain of the obtained signals into integer numbers. Figure 11 shows only detailed graphs of levels 1, 2, and 5, where defect signals experience a more drastic change than normal signals when the defect occurs.

The result can be an indicator that detects the location of defects. Since the defect is located in the center of the screw, the nut passes through the defective point at 1 and 3 seconds. Thus, defect vibrations occur at those times as shown in the graphs of levels 1 and 2. The high frequencies by the shock occurring from the defect position are in the range from 384 to $768 \mathrm{~Hz}$ and from 768 to $1,536 \mathrm{~Hz}$.
Also the amplitude can be an estimator of the defect progress. The continuous defect frequency, BPFS, is distributed around $58.7 \mathrm{~Hz}$ when the motor rotates with $399 \mathrm{rpm}$ in the ball-screw stage. The graph of level $5(48 \sim 96 \mathrm{~Hz})$ confirms that the component occurs over the whole time range with a certain magnitude of amplitude which tends to be changed by the progress of defect.

\section{Failure Diagnosis System}

A PC-based ball-screw failure diagnosis system was designed by applying the analysis of defect frequencies based on Wavelet transform, to detect and diagnose the defects of a ball-screw through the developed vibration monitoring method. The system is of stand-alone type; therefore, it can be applied to any equipment after modification of the diagnosis algorithm.

It has an accelerometer, 356A16, from PCB Piezotronics, a data acquisition, PXI-4472, from National Instruments (NI), and a signal analyzer, PXI-88187, from NI. The system was applied to a ball-screw-driven stage with motor, HFKP73, from Mitsubishi, ball-screw with the simulated defects, HTR 2020A, from ISSOKU, and linear motion (LM) guide mechanism, LP200-SC-600L-70-H-S, from LPK.

5.1. Failure Diagnosis Algorithm. As shown in Figure 12, the failure diagnosis system consists of signal acquisition, signal 


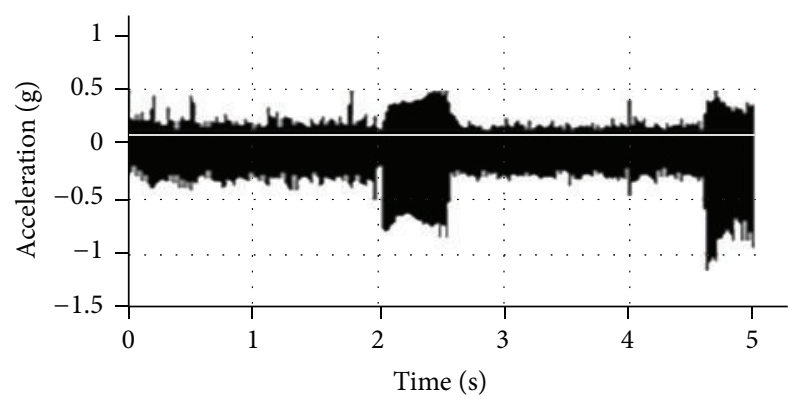

(a)

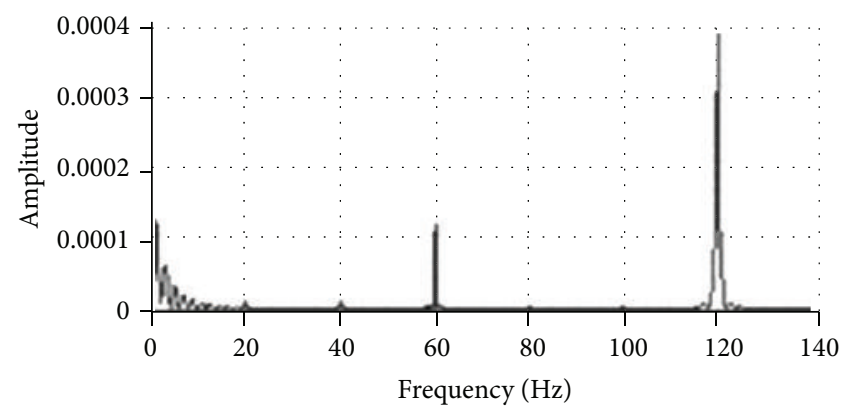

(b)

FIGURE 8: Frequency domain analysis of normal signal.
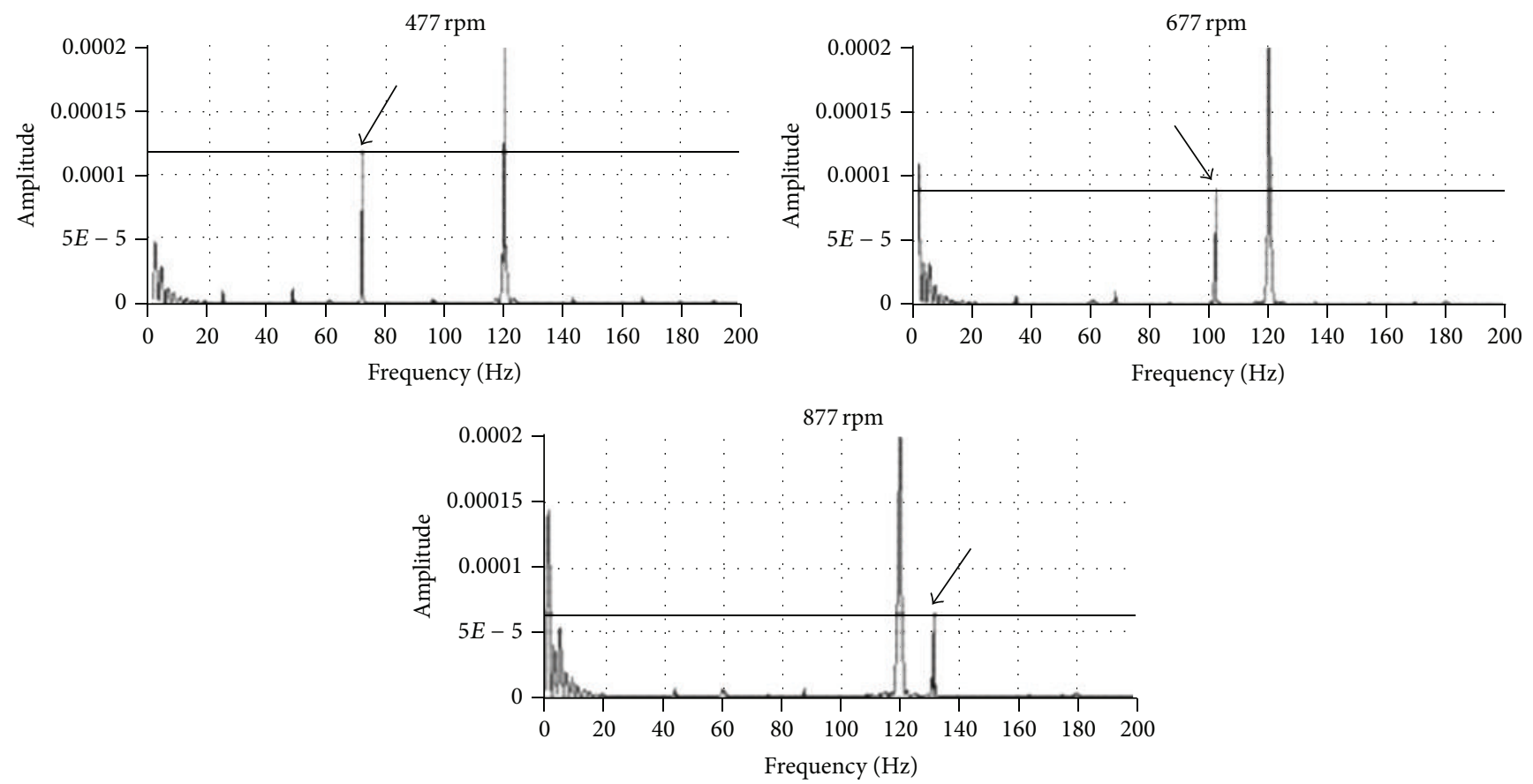

FIGURE 9: Defect frequency variation according to the rotating speed change of motor: the frequencies are indicated by arrows.

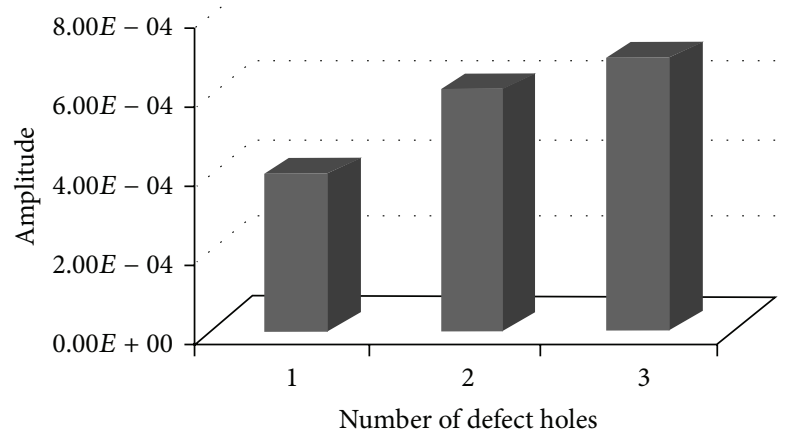

FIGURE 10: Defect amplitude variations according to the defect progress. 

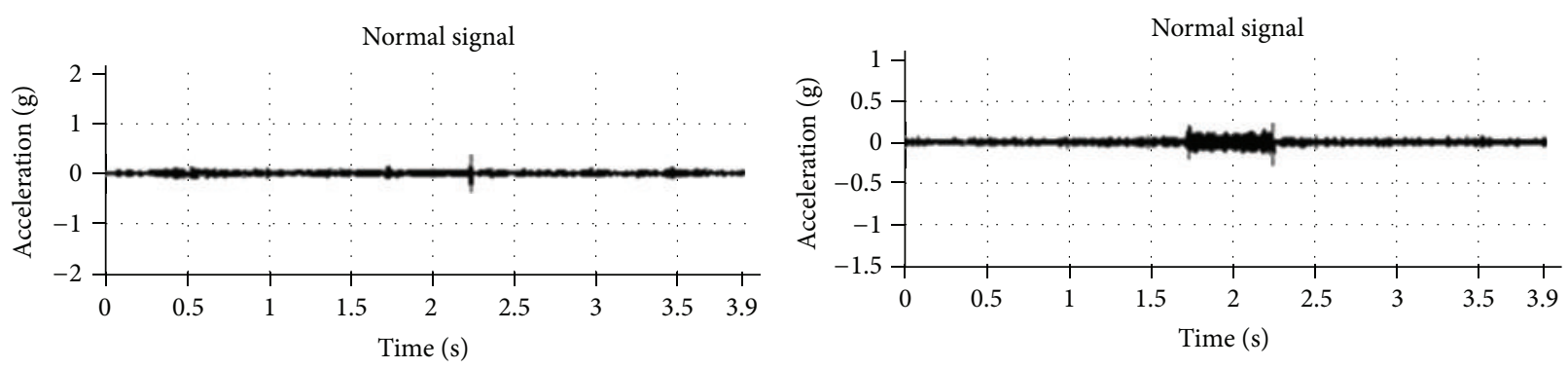

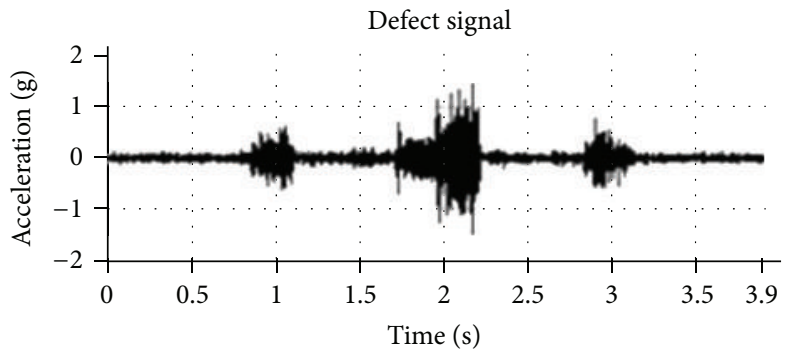

(a) Detail (level 1: 768 1536 Hz)

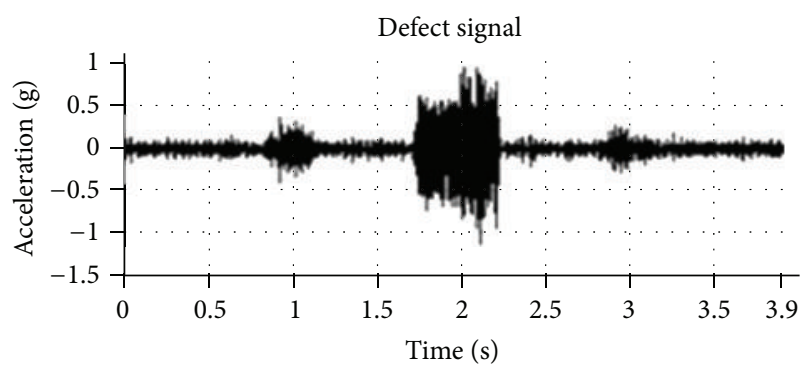

(b) Detail (level 2: 384 768 Hz)
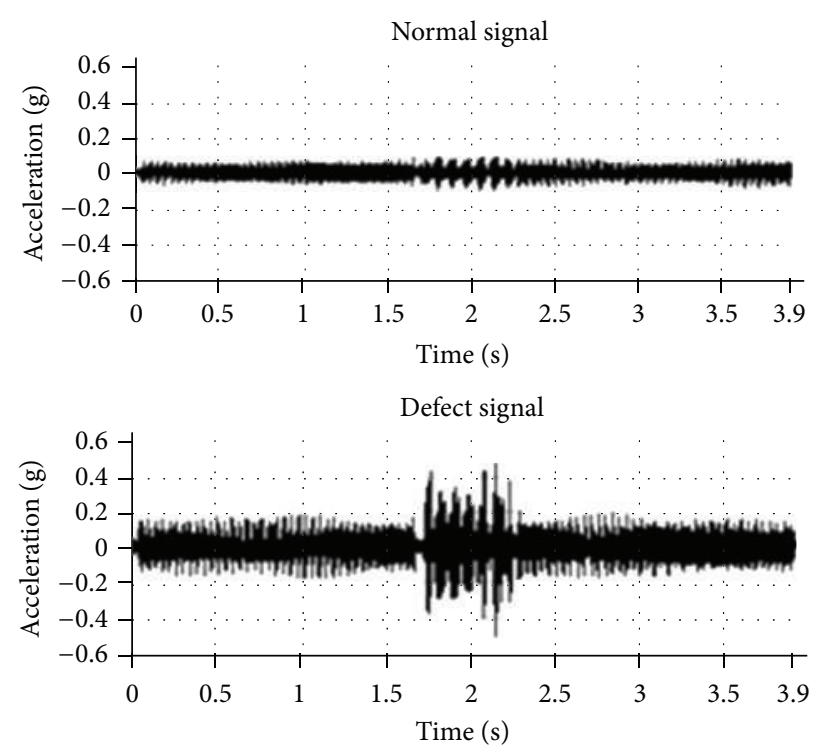

(c) Detail (level 5: 48 96 Hz)

FIGURE 11: Wavelet transform of normal and defect signals for one reciprocation of ball-screws during $4 \mathrm{~s}$ when the defect is at the center of screw shaft.

filtering, fault diagnosis, and saving modules. In particular, the delivered data is filtered using discrete Wavelet transform. The filtering module plays a role of band-pass filter for the range that includes the defect frequencies. The algorithm was implemented in a PC using graphic user interface (GUI) tool, LabVIEW 2010.

Figure 13 displays the interface screen of the developed failure diagnosis system. It monitors a certain frequency range of current condition. It also shows whether there is an initial defect and diagnoses defects by presenting a fault progress rate.

5.2. Failure Detection Test Results. We carried out a flaking failure diagnosis test for the ball-screw by using the designed failure diagnosis system. The vibration signals of the ballscrew-driven stage, which is under flaking failure, were collected during test. The failure diagnosis test results are shown in Figure 13.

The diagnosis of the flacking fatigue failure is performed under assumption that the weakened strength is synchronized with the progress of fatigue and this will result in large vibration. Taking into account the logarithmic progress of failure, the amplitude of normal signal was set as a min value and the amplitude of the maximum tolerable flaking was set as a max value. The amplitude in between the two was interpolated and then its progress was presented as percentage value. The result showed that approximately $65 \%$ of flaking failure has been progressed in Figure 13. It may 


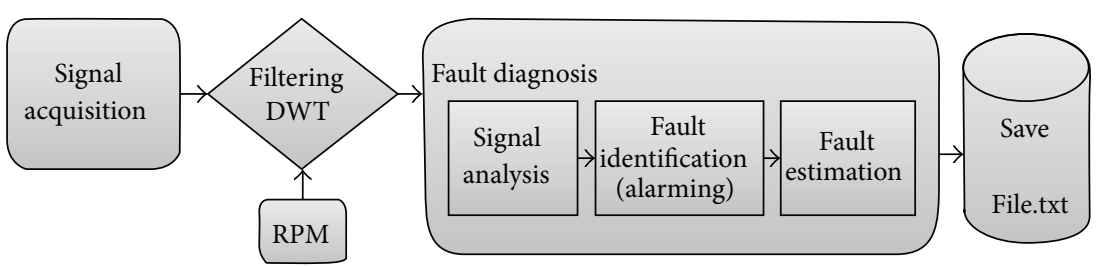

FIGURE 12: Scheme of failure diagnosis system.

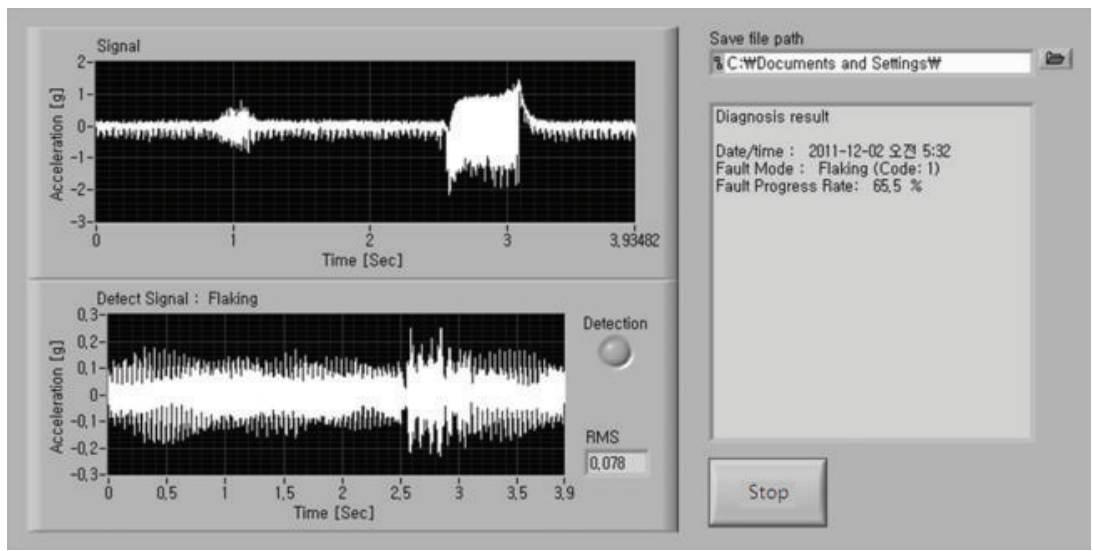

FIGURE 13: GUI and result of flaking failure diagnosis.

require verification by experts, because the estimation is based on the intuition of designers.

\section{Conclusion}

In this research, a condition monitoring method was developed by using a diagnosis system to detect and warn of failures in ball-screw-driven stage (Table 2). The system uses vibration as an indicator signal. A new analysis model is proposed to identify defect frequencies in ball-screw when it is operated continuously, that is, in steady state. Moreover, time-frequency analysis was carried out based on Wavelet transformation to detect the change of signal not only in steady state but also in transient condition. As a result, the following can be drawn:

(1) The defect frequencies in steady state can be derived by modeling ball-screw as rolling bearing, using relative speeds, rotational speed, diameter, and contact angle of each component. The frequencies are not integer times of the rotational speed of ball-screw so that they are easily distinguished from the harmonics of the speed. Because there is the larger amplitude of the defect vibration for more defects, it is effective to estimate the defect progress.

(2) In this study, defect frequencies are derived by assuming the ball-screw as a rolling bearing. Through use of the findings, we can detect both progression and occurrence time of failure in linearly reciprocating mechanical module.
(3) Wavelet transform shows the defect vibration clearly. In low frequency range, the defect frequency $58.7 \mathrm{~Hz}$ appeared. The amplitude of the frequency is used as estimator of the failure progress. In high frequency range, $768 \sim 1,536 \mathrm{~Hz}$, we were able to locate the defect position from the signal's abrupt change.

(4) Together with the identified frequency and Wavelet transform, we developed a failure diagnosis system. It can detect the defect and show the failure progress. It was set up in PC with stand-alone scheme; therefore, it can be applied easily to various equipment pieces.

\section{Nomenclature}

$\alpha$ : Contact angle

$D_{b}$ : Pitch diameter of ball of screw

$D_{i}$ : Diameter of screw groove

$D_{w}$ : Diameter of ball

$d_{e}$ : Diameter of nut groove

$d_{m}$ : Pitch diameter of ball

$d_{m}^{\prime}$ : Effective pitch diameter of ball

$L_{p}$ : Lead of screw

$n_{e}$ : Rotational speed of nut

$n_{i}$ : Rotational speed of screw

$v_{e}$ : Tangential linear speed of nut

$v_{i}$ : Tangential linear speed of screw

$v_{m}$ : Tangential linear speed of ball at its pitch circle

$z$ : Number of balls

$z^{\prime}$ : Effective number of balls. 
TABLE 2: Specifications of condition monitoring system.

\begin{tabular}{lccc}
\hline Equipment & Specifications & Value & Model (maker) \\
\hline \multirow{2}{*}{ Accelerometer } & Frequency range & $0.5-5000 \mathrm{~Hz}$ & $100 \mathrm{mV} / \mathrm{g}$ \\
& Voltage sensitivity & $24 \mathrm{bits}$ & $356 \mathrm{~A} 16$ (PCB Piezotronics) \\
& Resolution & $102.4 \mathrm{kS} / \mathrm{sec}$ & \\
A/D converter & Sampling rate & $\pm 10 \mathrm{~V}$ & PXI-4472 (National Instruments) \\
& Input range & & PXI-8187 (National Instruments) \\
\hline Signal analyzer & LabVIEW software (Ver. 2010)
\end{tabular}

\section{Conflict of Interests}

The authors declared no conflict of interests regarding publication of the paper.

\section{Acknowledgment}

This work was supported by the National Research Foundation of Korea (NRF) Grant funded by the Korea government (MSIP) (no. 2014R1A2A1A10052344).

\section{References}

[1] T. Wireman, World Class Maintenance Management, Industrial Press, New York, NY, USA, 1990.

[2] A. Ellis and A. Byron, "Condition based maintenance," The Jethro Project, vol. 10, pp. 1-5, 2008.

[3] A. K. S. Jardine, D. Lin, and D. Banjevic, "A review on machinery diagnostics and prognostics implementing condition-based maintenance," Mechanical Systems and Signal Processing, vol. 20, no. 7, pp. 1483-1510, 2006.

[4] R. K. Mobley, An Introduction to Predictive Maintenance, Butterworth-Heinemann, New York, NY, USA, 2nd edition, 1989.

[5] F. P. García Márquez, A. M. Tobias, J. M. Pinar Pérez, and M. Papaelias, "Condition monitoring of wind turbines: techniques and methods," Renewable Energy, vol. 46, pp. 169-178, 2012.

[6] J. K. Maslach, "Ferrographic analysis of grease-lubricated systems: an analysis of greases in roller bearings," Lubrication Engineering, vol. 52, no. 9, pp. 662-666, 1996.

[7] J. K. Spoerre, "Application of the cascade correlation algorithm (CCA) to bearing fault classification problems," Computers in Industry, vol. 32, no. 3, pp. 295-304, 1997.

[8] R. R. Schoen, T. G. Habetler, F. Kamran, and R. G. Bartheld, "Motor bearing damage detection using stator current monitoring," IEEE Transactions on Industry Applications, vol. 31, no. 6, pp. 1274-1279, 1995.

[9] R. K. Autar, "An automated diagnostic expert system for diesel engines," Journal of Engineering for Gas Turbines and Power, vol. 118, no. 3, pp. 673-679, 1996.

[10] C.-S. Liu, Fault detection of rolling element bearings [Ph.D. thesis], University of Washington, Seattle, Wash, USA, 2005.

[11] D.-S. Kim, S.-H. Lee, Y.-C. Yoo, and H.-E. Kim, "Life analysis through reliability test of ball screw for machine tools," in Proceedings of the Fall Conference of the Korean Society of Mechanical Engineers, pp. 1517-1521, KSMTE, Yongpyeong, Republic of Korea, November 2009 (Korean).
[12] T. A. Harris and J. I. McCool, "On the accuracy of rolling bearing fatigue life prediction," Journal of Tribology, vol. 118, no. 2, pp. 297-310, 1996.

[13] R. C. Juvinall and K. M. Marshek, Machine Component Design, John Wiley \& Sons, Hoboken, NJ, USA, 5th edition, 2011. 

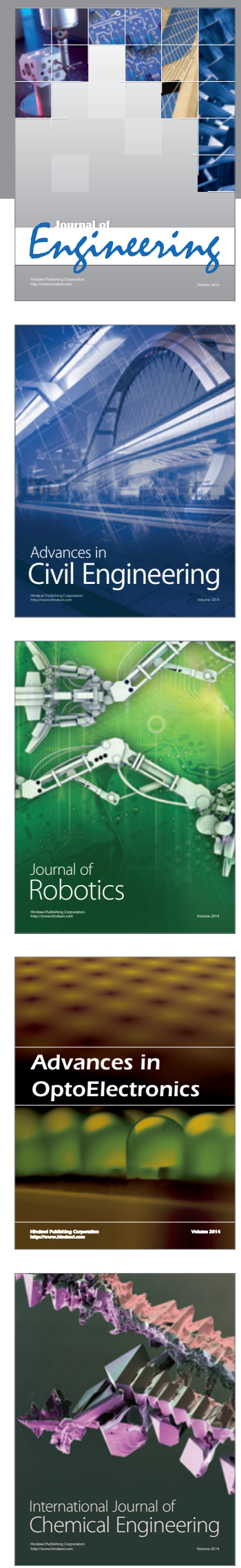

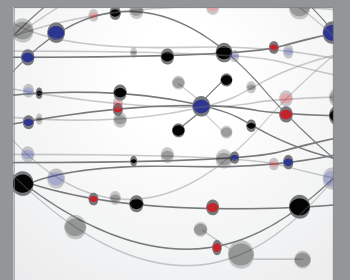

The Scientific World Journal
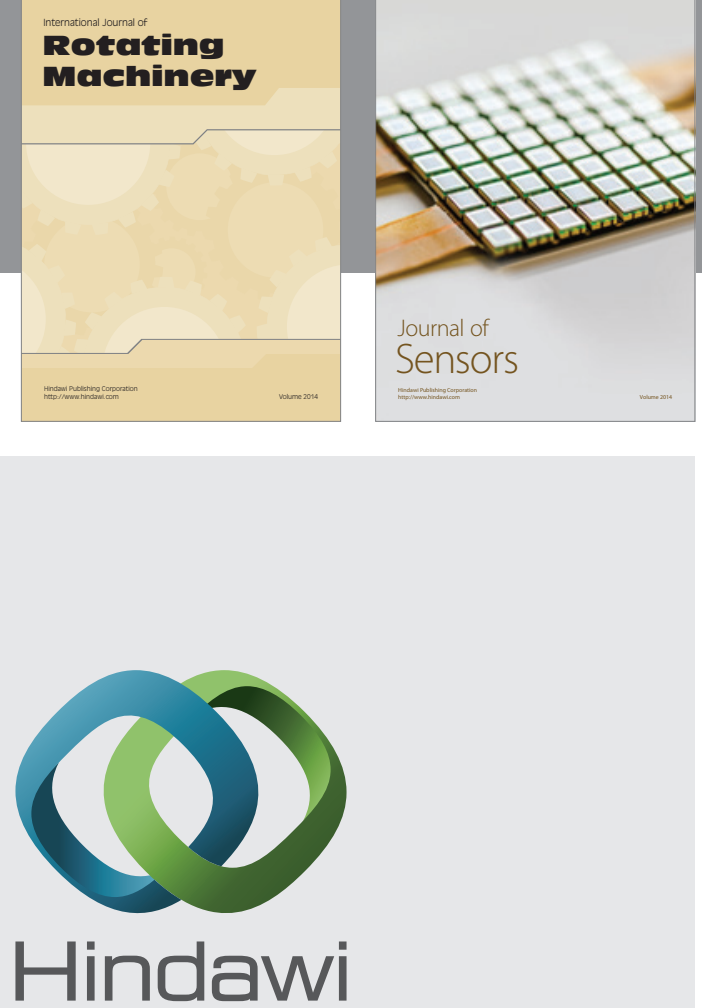

Submit your manuscripts at http://www.hindawi.com
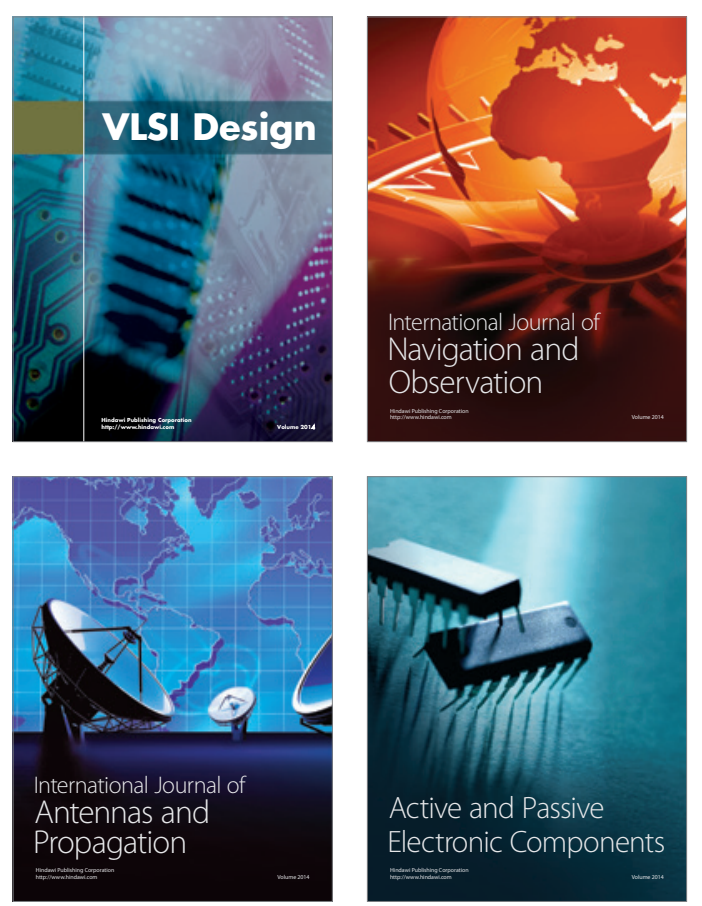
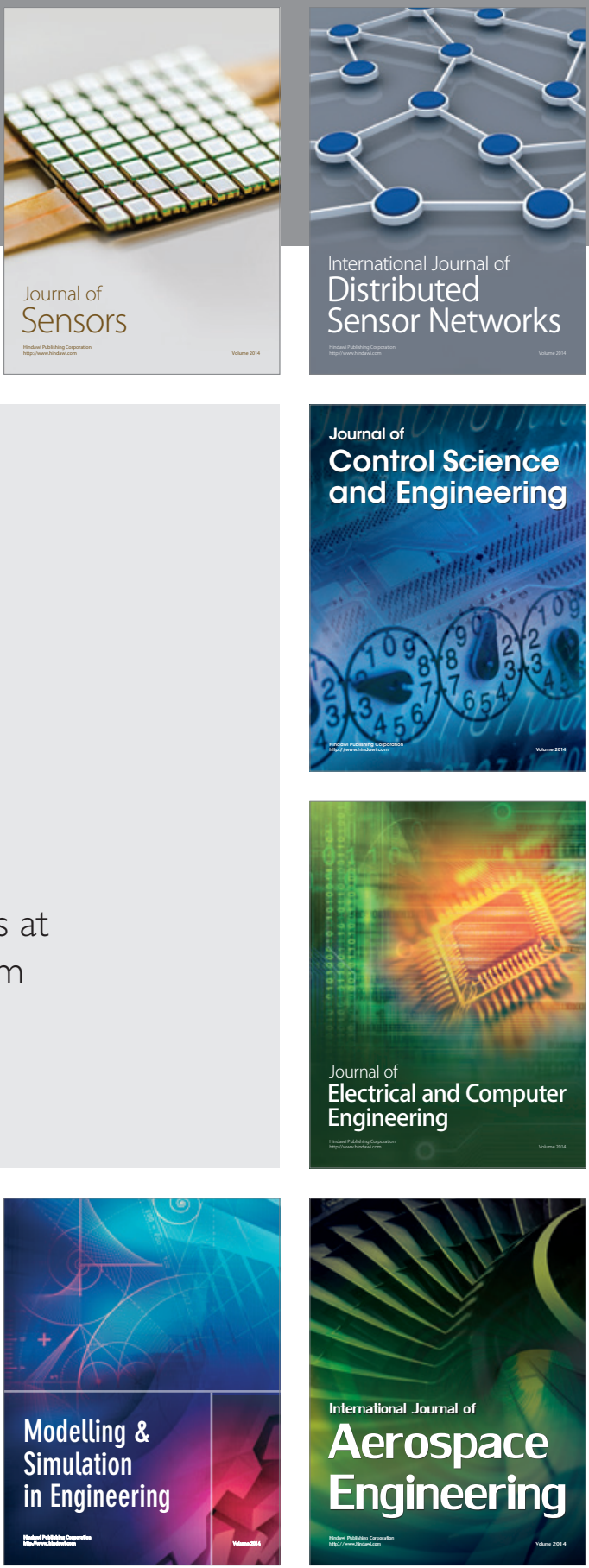

Journal of

Control Science

and Engineering
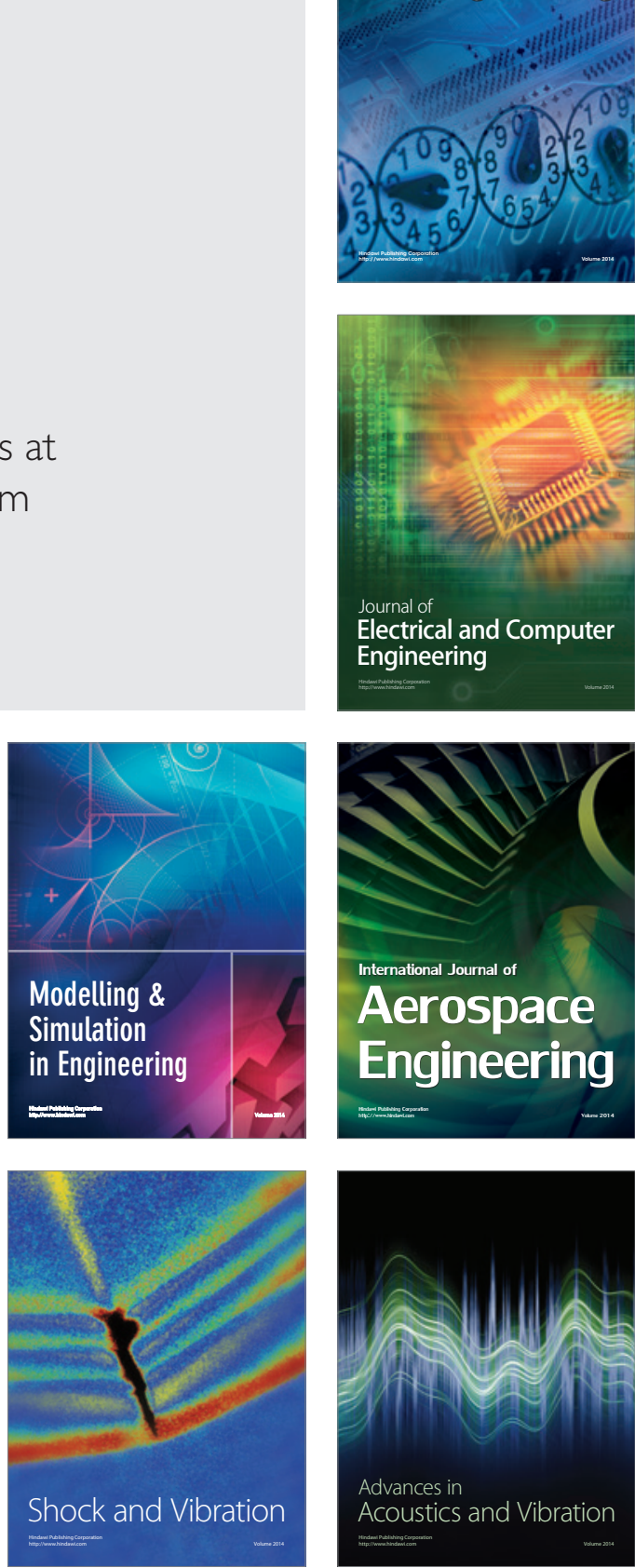